\title{
Strong and total Fenchel dualities for robust convex optimization problems
}

\author{
Mengdan Wang ${ }^{1}$, Donghui Fang ${ }^{1 *}$ and Zhe Chen ${ }^{2}$
}

\author{
"Correspondence: \\ dh_fang@jsu.edu.cn \\ ${ }^{1}$ College of Mathematics and \\ Statistics, Jishou University, Jishou, \\ 416000, P.R. China \\ Full list of author information is \\ available at the end of the article
}

\begin{abstract}
In this paper, we present some strong and total Fenchel dualities for convex programming problems with data uncertainty within the framework of robust optimization in locally convex Hausdorff vector spaces. By using the properties of the epigraph of the conjugate functions, we give some new constraint qualifications, which characterizes completely the strong duality and the stable strong duality. Moreover, some sufficient and/or necessary conditions for the total duality and converse duality are also obtained.
\end{abstract}

MSC: $90 C 25 ; 90 C 46$

Keywords: constraint qualification; strong duality; total duality; converse duality; robust optimization problems

\section{Introduction}

Let $X$ and $Y$ be real locally convex Hausdorff topological vector spaces, whose dual spaces, $X^{*}$ and $Y^{*}$, are endowed with the weak*-topologies $w^{*}\left(X^{*}, X\right)$ and $w^{*}\left(Y^{*}, Y\right)$, respectively. Let $f: X \rightarrow \overline{\mathbb{R}}:=\mathbb{R} \cup\{+\infty\}, g: Y \rightarrow \overline{\mathbb{R}}$ be proper convex functions, and let $A: X \rightarrow Y$ be a linear operator such that $A(\operatorname{dom} f) \cap \operatorname{dom} g \neq \emptyset$. The classical form of convex optimization problem in the absence of data uncertainty is (see, for example, [1-7])

$(\mathcal{P}) \quad \inf _{x \in X}\{f(x)+g(A x)\}$

and its Fenchel dual problem is

(D) $\sup _{y^{*} \in Y^{*}}\left\{-f^{*}\left(-A^{*} y^{*}\right)-g^{*}\left(y^{*}\right)\right\}$,

where $f^{*}$ and $g^{*}$ are the Fenchel conjugates of $f$ and $g$, respectively, and $A^{*}: Y^{*} \rightarrow X^{*}$ is the adjoint operator of $A$.

It is well known that the optimal values of these problems, $v(\mathcal{P})$ and $v(\mathcal{D})$, respectively, satisfy the so-called weak duality (i.e., $v(\mathcal{P}) \geq v(\mathcal{D})$ ), but a duality gap may occur (i.e., we may have $v(\mathcal{P})>v(\mathcal{D})$ ). A challenge in convex analysis has been to give sufficient conditions which guarantee the strong duality, that is, $v(\mathcal{P})=v(\mathcal{D})$ and $(\mathcal{D})$ has at least an optimal solution, and guarantee the converse strong duality, which corresponds to the situation in which $v(\mathcal{P})=v(\mathcal{D})$ and $(\mathcal{P})$ has at least an optimal solution (see, for instance, [1, 2, 5]). Several interiority-type conditions and epigraph type conditions have been given in order 
to establish the strong duality and the converse strong duality for the problem $(\mathcal{P})$ and problem $(\mathcal{D})$ in the literature ( $c f .[1,2,4-7]$ and the references therein). Especially, Li et al. established in [2] the strong duality and the converse strong duality in the most general setting, that is, $f$ and $g$ are not necessary lower semicontinuous (lsc) and $A$ is not necessary continuous.

Recently, the mathematical programming problems under uncertainty have received much attention ( $c f$. [8-21] and the references therein). The reason is the study of convex programming problems that are affected by data uncertainty is becoming increasingly important in optimization in many real-word optimization problems (cf. [11]). In particular, Li et al. considered the following uncertain convex programming problem (cf. [16]):

$$
(\tilde{P}) \inf _{x \in X} \sup _{\left(u_{1}, u_{2}\right) \in U_{1} \times U_{2}}\left\{f_{u_{1}}(x)+g_{u_{2}}(A x)\right\} .
$$

Under some additional assumptions, they established the strong duality between $(\tilde{P})$ and its dual problem.

Inspired by the works mentioned above, we continue to study the uncertain convex optimization problem $(\tilde{P})$ and the problem

$$
\text { (P) } \sup _{\left(u_{1}, u_{2}\right) \in U_{1} \times U_{2}} \inf _{x \in X}\left\{f_{u_{1}}(x)+g_{u_{2}}(A x)\right\} \text {. }
$$

Unlike in [16], we assume in this paper that $f_{u_{1}}: X \rightarrow \overline{\mathbb{R}}, u_{1} \in U_{1}$ and $g_{u_{2}}: Y \rightarrow \overline{\mathbb{R}}, u_{2} \in U_{2}$ are proper convex functions (not necessarily lsc), $A: X \rightarrow Y$ is a linear operator, and $U_{1}$, $U_{2}$ are subsets of a locally convex space $Z$. Following [16], we define the dual problem by

(D) $\sup _{\left(u_{1}, u_{2}\right) \in U_{1} \times U_{2}} \sup _{y^{*} \in Y^{*}}\left\{-g_{u_{2}}^{*}\left(y^{*}\right)-f_{u_{1}}^{*}\left(-A^{*} y^{*}\right)\right\}$.

In particular, in the case when $U_{1}$ and $U_{2}$ are singletons, problems $(\tilde{P})$ and $(P)$ coincide with the problem $(\mathcal{P})$.

Let $v(P), v(\tilde{P})$ and $v(D)$ denote the optimal values of problems $(P),(\tilde{P})$ and $(D)$, respectively. Obviously, $v(D) \leq v(P) \leq v(\tilde{P})$, that is, the weak dualities hold between $(P)$ and $(D)$ and between $(\tilde{P})$ and $(D)$. Our main aim in the present paper is to give some new regularity conditions which completely characterize the strong dualities and the stable strong dualities between $(P)$ and $(D)$ and between $(\tilde{P})$ and $(D)$, and provide sufficient and/or necessary conditions for the total duality, the converse dualities between $(P)$ and $(D)$. Most results obtained in this paper seem new and are proper extensions of the known results in $[2,16]$; in particular, our Theorem 3.3 extends and improves the result in [16], Theorem 5.1, and, even in the special case when $U_{1}$ and $U_{2}$ are singletons, our Corollary 5.1 improves the corresponding result in [2], Theorem 6.9.

This paper is organized as follows. The next section contains some necessary notations and preliminary results. In Section 3, we give some new regularity conditions which completely characterize the strong dualities and the stable strong dualities between $(P)$ and $(D)$ and between $(\tilde{P})$ and $(D)$. Some sufficient and/or necessary conditions for the total duality and the converse duality between $(P)$ and $(D)$ are given in Sections 4 and 5 , respectively. 


\section{Notations and preliminary results}

The notations used in the present paper are standard ( $c f$. [3]). In particular, we assume throughout the whole paper that $X$ and $Y$ are real locally convex Hausdorff topological vector spaces, and let $X^{*}$ denote the dual space of $X$, endowed with the weak*-topology $w^{*}\left(X^{*}, X\right)$. By $\left\langle x^{*}, x\right\rangle$, we shall denote the value of the functional $x^{*} \in X^{*}$ at $x \in X$; i.e., $\left\langle x^{*}, x\right\rangle=x^{*}(x)$. Let $Z \subset X$, the interior, closure, convex hull, and the convex conical hull of $Z$ are denoted by int $Z, \operatorname{cl} Z, \operatorname{co} Z$, and cone $Z$, respectively. If $W \subseteq X^{*}$, then $\mathrm{cl} W$ denotes the weak*-closure of $W$. For the whole paper, we endow $X^{*} \times \mathbb{R}$ with the product topology of $w^{*}\left(X^{*}, X\right)$ and the usual Euclidean topology.

The indicator function $\delta_{Z}: X \rightarrow \overline{\mathbb{R}}$ of the nonempty set $Z$ is defined by

$$
\delta_{Z}(x):= \begin{cases}0, & x \in Z \\ +\infty, & \text { otherwise }\end{cases}
$$

Let $f: X \rightarrow \overline{\mathbb{R}}$ be a proper convex function. The effective domain, the epigraph and the conjugate function of $f$ are defined, respectively, by

$$
\begin{aligned}
& \operatorname{dom} f:=\{x \in X: f(x)<+\infty\}, \\
& \text { epif }:=\{(x, r) \in X \times \mathbb{R}: f(x) \leq r\}
\end{aligned}
$$

and

$$
f^{*}\left(x^{*}\right):=\sup \left\{\left\langle x^{*}, x\right\rangle-f(x): x \in X\right\} \quad \text { for each } x^{*} \in X^{*}
$$

By definition, the Young-Fenchel inequality holds:

$$
f(x)+f^{*}\left(x^{*}\right) \geq\left\langle x, x^{*}\right\rangle \text { for each pair }\left(x, x^{*}\right) \in X \times X^{*} .
$$

The lsc hull and the lsc convex hull of $f$, denoted respectively by $\operatorname{cl} f$ and $\operatorname{cl}(\operatorname{co} f)$, are defined by

$$
\operatorname{epi}(\operatorname{cl} f)=\operatorname{cl}(\text { epi } f) \quad \text { and } \quad \operatorname{epi}(\operatorname{cl}(\operatorname{co} f))=\operatorname{cl}[\operatorname{co}(\operatorname{epi} f)]
$$

If $\mathrm{cl} f$ is proper, then the following equality holds (cf. [3]):

$$
f^{* *}=\operatorname{cl} f \text {. }
$$

If $g, h$ are proper, then

$$
\text { epi } g^{*}+\text { epi } h^{*} \subseteq \operatorname{epi}(g+h)^{*}
$$

and

$$
g \leq h \quad \Rightarrow \quad g^{*} \geq h^{*} \quad \Leftrightarrow \quad \text { epi } g^{*} \subseteq \text { epi } h^{*} .
$$


In particular, let $p \in X^{*}$. Define a function on $X$ that $p(x):=\langle p, x\rangle$ for each $x \in X$. Then, for any $a \in \mathbb{R}$ and any function $h: X \rightarrow \overline{\mathbb{R}}$,

$$
\begin{aligned}
& (h+p+a)^{*}\left(x^{*}\right)=h^{*}\left(x^{*}-p\right)-a \quad \text { for each } x^{*} \in X^{*} ; \\
& \operatorname{epi}(h+p+a)^{*}=\operatorname{epi} h^{*}+(p,-a) .
\end{aligned}
$$

Let $x \in \operatorname{dom} f$. The subdifferential of $f$ at $x$ is the convex set defined by

$$
\partial f(x):=\left\{x^{*} \in X^{*}: f(x)+\left\langle x^{*}, y-x\right\rangle \leq f(y) \text { for all } y \in X\right\} .
$$

Then, by definitions,

$$
0 \in \partial f(x) \quad \Leftrightarrow \quad x \text { is a minimizer of } f
$$

and

$$
\left(x^{*},\left\langle x^{*}, x\right\rangle-f(x)\right) \in \text { epif }^{*} \quad \text { for all } x^{*} \in \partial f(x) .
$$

Moreover, by [3], Theorem 2.4.2(iii), the Young equality holds

$$
f(x)+f^{*}\left(x^{*}\right)=\left\langle x^{*}, x\right\rangle \quad \Leftrightarrow \quad x^{*} \in \partial f(x) .
$$

For functions $g, h: X \rightarrow \overline{\mathbb{R}}$, we define the infimal convolution of $g$ and $h$ as the function $g \square h: X \rightarrow \mathbb{R} \cup\{ \pm \infty\}$ given by

$$
(g \square h)(a):=\inf _{x \in X}\{g(x)+h(a-x)\} .
$$

If $g$ and $h$ are lsc and $\operatorname{dom} g \cap \operatorname{dom} h \neq \emptyset$, then by [3] we have that

$$
(g \square h)^{*}=g^{*}+h^{*}, \quad(g+h)^{*}=\operatorname{cl}\left(g^{*} \square h^{*}\right)
$$

and

$$
\text { epi } g^{*}+\text { epi } h^{*} \subseteq \operatorname{epi}\left(g^{*} \square h^{*}\right) \subseteq \operatorname{cl}\left(\text { epi } g^{*}+\text { epi } h^{*}\right)
$$

\section{Robust stable Fenchel duality}

Let $X, Y$ and $Z$ be real locally convex Hausdorff topological vector spaces, $U_{1}$ and $U_{2}$ be subsets of $Z$. Let $f_{u_{1}}: X \rightarrow \overline{\mathbb{R}}, u_{1} \in U_{1}$, and $g_{u_{2}}: Y \rightarrow \overline{\mathbb{R}}, u_{2} \in U_{2}$ be proper convex functions, $A: X \rightarrow Y$ be a linear operator such that $\bigcap_{\left(u_{1}, u_{2}\right) \in U_{1} \times U_{2}}\left[A\left(\operatorname{dom} f_{u_{1}}\right) \cap\left(\operatorname{dom} g_{u_{2}}\right)\right] \neq \emptyset$. For simplicity, we denote

$$
u:=\left(u_{1}, u_{2}\right) \quad \text { and } \quad U:=U_{1} \times U_{2} .
$$

We shall consider the identity map id $\operatorname{id}_{\mathbb{R}}$ on $\mathbb{R}$, and the image set $\left(A^{*} \times \mathrm{id}_{\mathbb{R}}\right)\left(\right.$ epi $\left.g_{u_{2}}^{*}\right)$ of epi $g_{u_{2}}^{*}$ through the map $A^{*} \times \mathrm{id}_{\mathbb{R}}: Y^{*} \times \mathbb{R} \rightarrow X^{*} \times \mathbb{R}$, that is,

$$
\left(x^{*}, r\right) \in\left(A^{*} \times \operatorname{id}_{\mathbb{R}}\right)\left(\text { epi } g_{u_{2}}^{*}\right) \Leftrightarrow\left\{\begin{array}{l}
\exists y^{*} \in Y^{*} \text { such that }\left(y^{*}, r\right) \in \operatorname{epi} g_{u_{2}}^{*} \\
\text { and } A^{*} y^{*}=x^{*}
\end{array}\right.
$$


Given $p \in X^{*}$, consider the following robust optimization problems with a linear perturbation:

$$
\left(P_{p}\right) \quad \sup _{u \in U} \inf _{x \in X}\left\{f_{u_{1}}(x)+g_{u_{2}}(A x)-\langle p, x\rangle\right\}
$$

and

$$
\left(\tilde{P}_{p}\right) \quad \inf _{x \in X} \sup _{u \in U}\left\{f_{u_{1}}(x)+g_{u_{2}}(A x)-\langle p, x\rangle\right\} .
$$

We define the corresponding Fenchel dual problem by

$$
\left(D_{p}\right) \quad \sup _{u \in U} \sup _{y^{*} \in Y^{*}}\left\{-g_{u_{2}}^{*}\left(y^{*}\right)-f_{u_{1}}^{*}\left(p-A^{*} y^{*}\right)\right\}
$$

As usual, we use $v\left(P_{p}\right), v\left(\tilde{P}_{p}\right)$ and $v\left(D_{p}\right)$ to stand for the optimal values of the problems $\left(P_{p}\right),\left(\tilde{P}_{p}\right)$ and $\left(D_{p}\right)$, respectively, that is,

$$
\begin{aligned}
& v\left(P_{p}\right):=\sup _{u \in U} \inf _{x \in X}\left\{f_{u_{1}}(x)+g_{u_{2}}(A x)-\langle p, x\rangle\right\}, \\
& v\left(\tilde{P}_{p}\right):=\inf _{x \in X} \sup _{u \in U}\left\{f_{u_{1}}(x)+g_{u_{2}}(A x)-\langle p, x\rangle\right\},
\end{aligned}
$$

and

$$
v\left(D_{p}\right):=\sup _{u \in U} \sup _{y^{*} \in Y^{*}}\left\{-g_{u_{2}}^{*}\left(y^{*}\right)-f_{u_{1}}^{*}\left(p-A^{*} y^{*}\right)\right\}
$$

By definitions, it is easy to check that the following inequalities hold:

$$
v\left(D_{p}\right) \leq v\left(P_{p}\right) \leq v\left(\tilde{P}_{p}\right) \quad \text { for each } p \in X^{*} .
$$

Moreover, by definitions, we can get that

$$
v\left(P_{p}\right)=\sup _{u \in U}\left[-\left(f_{u_{1}}+g_{u_{2}} \circ A\right)^{*}(p)\right]=-\inf _{u \in U}\left(f_{u_{1}}+g_{u_{2}} \circ A\right)^{*}(p)
$$

and

$$
v\left(D_{p}\right)=\sup _{u \in U}\left[-\left(f_{u_{1}}^{*} \square A^{*} g_{u_{2}}^{*}\right)(p)\right]=-\inf _{u \in U}\left(f_{u_{1}}^{*} \square A^{*} g_{u_{2}}^{*}\right)(p) .
$$

In particular, in the case when $p=0$, problems $\left(P_{p}\right),\left(\tilde{P}_{p}\right)$ and $\left(D_{p}\right)$ are just problems $(P)$, $(\tilde{P})$ and $(D)$, which are defined by (1.4), (1.3) and (1.5), respectively. Furthermore, by (3.4) and (3.5), the following equalities hold:

$$
v(P)=-\inf _{u \in U}\left(f_{u_{1}}+g_{u_{2}} \circ A\right)^{*}(0),
$$

and

$$
v(D)=-\inf _{u \in U}\left(f_{u_{1}}^{*} \square A^{*} g_{u_{2}}^{*}\right)(0) .
$$


This section is devoted to the study of the strong dualities and stable strong dualities between $(P)$ and $(D)$ and between $(\tilde{P})$ and $(D)$, which are defined as follows.

Definition 3.1 It is said that

(i) the strong duality holds between $(P)$ and $(D)$ (resp., between $(\tilde{P})$ and $(D))$ if $v(P)=v(D)($ resp., $v(\tilde{P})=v(D))$ and $(D)$ has an optimal solution;

(ii) the stable strong duality holds between $(P)$ and $(D)$ (resp., between $(\tilde{P})$ and $(D))$ if for each $p \in X^{*}$, the strong duality holds between $\left(P_{p}\right)$ and $\left(D_{p}\right)$ (resp., between $\left(\tilde{P}_{p}\right)$ and $\left.\left(D_{p}\right)\right)$.

Remark 3.1 Clearly, if the strong duality holds between $\left(\tilde{P}_{p}\right)$ and $\left(D_{p}\right)$, by (3.3), then the strong duality holds between $\left(P_{p}\right)$ and $\left(D_{p}\right)$. However, the converse is not true in general.

Definition 3.2 The family $\left(f_{u_{1}}, g_{u_{2}} ; A ; U\right)$ is said to satisfy

(a) the strong further regularity condition $(S F R C)$ if

$$
\begin{aligned}
& \operatorname{cl}\left[\operatorname{co} \bigcup_{u \in U} \operatorname{epi}\left(f_{u_{1}}+g_{u_{2}} \circ A\right)^{*}\right] \cap(\{0\} \times \mathbb{R}) \\
& \subseteq \subseteq \bigcup_{u \in U}\left(\operatorname{epi} f_{u_{1}}^{*}+\left(A^{*} \times \operatorname{id} \mathbb{R}_{\mathbb{R}}\right)\left(\operatorname{epi} g_{u_{2}}^{*}\right)\right) \cap(\{0\} \times \mathbb{R})
\end{aligned}
$$

(b) the asymptotic further regularity condition $(A F R C)$ if

$$
\begin{aligned}
& \operatorname{cl}\left[\bigcup_{u \in U} \operatorname{epi}\left(f_{u_{1}}+g_{u_{2}} \circ A\right)^{*}\right] \cap(\{0\} \times \mathbb{R}) \\
& \subseteq \subseteq \bigcup_{u \in U}\left(\operatorname{epi} f_{u_{1}}^{*}+\left(A^{*} \times \operatorname{id}_{\mathbb{R}}\right)\left(\operatorname{epi} g_{u_{2}}^{*}\right)\right) \cap(\{0\} \times \mathbb{R}) ;
\end{aligned}
$$

(c) the further regularity condition $(F R C)$ if

$$
\begin{aligned}
& \bigcup_{u \in U} \operatorname{epi}\left(f_{u_{1}}+g_{u_{2}} \circ A\right)^{*} \cap(\{0\} \times \mathbb{R}) \\
& \subseteq \bigcup_{u \in U}\left(\operatorname{epi} f_{u_{1}}^{*}+\left(A^{*} \times \operatorname{id}_{\mathbb{R}}\right)\left(\operatorname{epi} g_{u_{2}}^{*}\right)\right) \cap(\{0\} \times \mathbb{R})
\end{aligned}
$$

(d) the strong closure condition $(S C C)$ if

$$
\operatorname{cl}\left[\operatorname{co} \bigcup_{u \in U} \operatorname{epi}\left(f_{u_{1}}+g_{u_{2}} \circ A\right)^{*}\right] \subseteq \bigcup_{u \in U}\left(\operatorname{epi} f_{u_{1}}^{*}+\left(A^{*} \times \operatorname{id} \mathbb{R}_{\mathbb{R}}\right)\left(\operatorname{epi} g_{u_{2}}^{*}\right)\right)
$$

(e) the asymptotic closure condition $(A C C)$ if

$$
\mathrm{cl}\left[\bigcup_{u \in U} \operatorname{epi}\left(f_{u_{1}}+g_{u_{2}} \circ A\right)^{*}\right] \subseteq \bigcup_{u \in U}\left(\operatorname{epif} f_{u_{1}}^{*}+\left(A^{*} \times \operatorname{id} \mathbb{R}_{\mathbb{R}}\right)\left(\operatorname{epi} g_{u_{2}}^{*}\right)\right) ;
$$

(f) the closure condition $(C C)$ if

$$
\bigcup_{u \in U} \operatorname{epi}\left(f_{u_{1}}+g_{u_{2}} \circ A\right)^{*} \subseteq \bigcup_{u \in U}\left(\operatorname{epi} f_{u_{1}}^{*}+\left(A^{*} \times \operatorname{id} \mathbb{R}_{\mathbb{R}}\right)\left(\operatorname{epi} g_{u_{2}}^{*}\right)\right)
$$


Remark 3.2 (i) Note from [2], Lemma 3.2, that

$$
\operatorname{epi} f_{u_{1}}^{*}+\left(A^{*} \times \operatorname{id} \mathbb{R}_{\mathbb{R}}\right)\left(\operatorname{epi} g_{u_{2}}^{*}\right) \subseteq \operatorname{epi}\left(f_{u_{1}}+g_{u_{2}} \circ A\right)^{*} \quad \text { for each } u \in U \text {. }
$$

It follows that

$$
\begin{aligned}
\bigcup_{u \in U}\left(\operatorname{epi} f_{u_{1}}^{*}+\left(A^{*} \times \operatorname{id}_{\mathbb{R}}\right)\left(\operatorname{epi} g_{u_{2}}^{*}\right)\right) & \subseteq \bigcup_{u \in U} \operatorname{epi}\left(f_{u_{1}}+g_{u_{2}} \circ A\right)^{*} \\
& \subseteq \operatorname{cl}\left[\bigcup_{u \in U} \operatorname{epi}\left(f_{u_{1}}+g_{u_{2}} \circ A\right)^{*}\right] \\
& \subseteq \operatorname{cl}\left[\operatorname{co} \bigcup_{u \in U} \operatorname{epi}\left(f_{u_{1}}+g_{u_{2}} \circ A\right)^{*}\right]
\end{aligned}
$$

Thus, the inclusions in (3.7)-(3.12) can be replaced by equalities. Moreover, by (3.13), we see that

$$
(S F R C) \Rightarrow(A F R C) \Rightarrow(F R C)
$$

and

$$
(S C C) \Rightarrow(A C C) \Rightarrow(C C) .
$$

(ii) Recall from [2], Definition 3.1, that the triple $(f, g ; A)$ satisfies the $(C C)_{A}$ if

$$
\operatorname{epi}(f+g \circ A)^{*}=\operatorname{epi} f^{*}+\left(A^{*} \times \operatorname{id} \mathbb{R}_{\mathbb{R}}\right)\left(\text { epi } g^{*}\right)
$$

and from [2], Definition 4.2, that the triple $(f, g ; A)$ satisfies the $(F R C)_{A}$ if

$$
(f+g \circ A)^{*}(0) \geq\left(f^{*} \square A^{*} g^{*}\right)(0),
$$

and there exists $x^{*} \in X^{*}$ such that $\left(f^{*} \square A^{*} g^{*}\right)(0)=f^{*}\left(-x^{*}\right)+\left(A^{*} g^{*}\right)\left(x^{*}\right)$ and the infimum in the definition of $\left(A^{*} g^{*}\right)\left(x^{*}\right)$ is attained, where $f^{*} \square A^{*} g^{*}$ denotes the infimal convolution of $f^{*}$ and $A^{*} g^{*}$. By [2], Proposition 4.3, the $(F R C)_{A}$ for the triple $(f, g ; A)$ is equivalent to

$$
\operatorname{epi}(f+g \circ A)^{*} \cap(\{0\} \times \mathbb{R}) \subseteq\left(\text { epi } f^{*}+\left(A^{*} \times \operatorname{id} \mathbb{R}\right)\left(\text { epi } g^{*}\right)\right) \cap(\{0\} \times \mathbb{R}) .
$$

Thus, in the case when $U_{1}$ and $U_{2}$ are singletons, (SFRC), (AFRC) and (FRC) are same and turned into $(F R C)_{A}$ for the triple $(f, g ; A)$; meanwhile, $(S C C),(A C C)$ and $(C C)$ for the triple $\left(f_{u_{1}}, g_{u_{2}} ; A ; U\right)$ are same as $(C C)_{A}$ for the triple $(f, g ; A)$.

The following proposition describes the relationship between the $(S C C)$ (resp., the $(A C C)$, the $(C C))$ and the $(S F R C)$ (resp., the $(A F R C)$, the $(F R C))$.

Proposition 3.1 The family $\left(f_{u_{1}}, g_{u_{2}} ; A ; U\right)$ satisfies the (SCC) (resp., the (ACC), the $(C C)$ ) if and only iffor each $p \in X^{*},\left(f_{u_{1}}-p, g_{u_{2}} ; A ; U\right)$ satisfies the (SFRC) (resp., the (AFRC), the $(F R C)$ ). 
Proof Let $p \in X^{*}$ and let $K_{1}(p), K_{2}(p)$ be defined by

$$
K_{1}(p):=\bigcup_{u \in U} \operatorname{epi}\left(f_{u_{1}}-p+g_{u_{2}} \circ A\right)^{*}
$$

and

$$
K_{2}(p):=\bigcup_{u \in U}\left(\operatorname{epi}\left(f_{u_{1}}-p\right)^{*}+\left(A^{*} \times \operatorname{id}_{\mathbb{R}}\right)\left(\operatorname{epi} g_{u_{2}}^{*}\right)\right)
$$

respectively. Then, by (2.2), the following equalities are clear:

$$
K_{1}(p)=K_{1}(0)+(-p, 0) \quad \text { and } \quad K_{2}(p)=K_{2}(0)+(-p, 0) .
$$

Hence, we have that

$$
K_{1}(p) \cap(\{0\} \times \mathbb{R})=K_{1}(0) \cap(\{p\} \times \mathbb{R})+(-p, 0)
$$

and

$$
K_{2}(p) \cap(\{0\} \times \mathbb{R})=K_{2}(0) \cap(\{p\} \times \mathbb{R})+(-p, 0) .
$$

Thus, the conclusion holds by definitions, and the proof is complete.

Lemma 3.1 Let $r \in \mathbb{R}$. Then the following assertions hold:

(i) $(p, r) \in \bigcup_{u \in U}\left(\right.$ epi $f_{u_{1}}^{*}+\left(A^{*} \times \mathrm{id}_{\mathbb{R}}\right)\left(\right.$ epi $\left.\left.g_{u_{2}}^{*}\right)\right)$ if and only if there exist $\bar{u}=\left(\bar{u}_{1}, \bar{u}_{2}\right) \in U$ and $y^{*} \in Y^{*}$ satisfying

$$
f_{\bar{u}_{1}}^{*}\left(p-A^{*} y^{*}\right)+g_{\bar{u}_{2}}^{*}\left(y^{*}\right) \leq r .
$$

(ii) Suppose that

$$
f_{u_{1}}: X \rightarrow \overline{\mathbb{R}}, \quad u_{1} \in U_{1}, \quad g_{u_{2}}: Y \rightarrow \overline{\mathbb{R}}, \quad u_{2} \in U_{2}
$$

are lsc and $A$ is continuous.

Then $(p, r) \in \operatorname{cl}\left[\operatorname{co} \bigcup_{u \in U} \operatorname{epi}\left(f_{u_{1}}+g_{u_{2}} \circ A\right)^{*}\right]$ if and only if $v\left(\tilde{P}_{p}\right) \geq-r$.

Proof Without loss of generality, we assume that $p=0$.

(i) Let $(0, r) \in \bigcup_{u \in U}\left(\right.$ epi $f_{u_{1}}^{*}+\left(A^{*} \times \operatorname{id}_{\mathbb{R}}\right)\left(\right.$ epi $\left.\left.g_{u_{2}}^{*}\right)\right)$. Then there exists $\bar{u}=\left(\bar{u}_{1}, \bar{u}_{2}\right) \in U$ such that $(0, r) \in\left(\operatorname{epi} f_{\bar{u}_{1}}^{*}+\left(A^{*} \times \operatorname{id} \mathbb{R}_{\mathbb{R}}\right)\left(\operatorname{epi} g_{\bar{u}_{2}}^{*}\right)\right)$. Thus, there exist $\left(x_{1}^{*}, r_{1}\right) \in \operatorname{epi} f_{\bar{u}_{1}}^{*}$ and $\left(x_{2}^{*}, r_{2}\right) \in$ $\left(A^{*} \times \mathrm{id}_{\mathbb{R}}\right)\left(\right.$ epi $\left.g_{\bar{u}_{2}}^{*}\right)$ such that

$$
x_{1}^{*}+x_{2}^{*}=0 \text { and } r_{1}+r_{2}=r .
$$

Moreover, there exists $\bar{y}^{*} \in Y^{*}$ such that $A^{*} \bar{y}^{*}=x_{2}^{*}$ and $\left(\bar{y}^{*}, r_{2}\right) \in$ epi $g_{\bar{u}_{2}}^{*}$. Consequently, $f_{\bar{u}_{1}}^{*}\left(x_{1}^{*}\right) \leq r_{1}$ and $g_{\bar{u}_{2}}^{*}\left(\bar{y}^{*}\right) \leq r_{2}$. This together with (3.15) implies that

$$
f_{\bar{u}_{1}}^{*}\left(-A^{*} y^{*}\right)+g_{\bar{u}_{2}}^{*}\left(y^{*}\right)=f_{\bar{u}_{1}}^{*}\left(x_{1}^{*}\right)+g_{\bar{u}_{2}}^{*}\left(\bar{y}^{*}\right) \leq r_{1}+r_{2}=r .
$$


Conversely, suppose that there exist $\bar{u}=\left(\bar{u}_{1}, \bar{u}_{2}\right) \in U$ and $y^{*} \in Y^{*}$ satisfying

$$
f_{\bar{u}_{1}}^{*}\left(-A^{*} y^{*}\right)+g_{\bar{u}_{2}}^{*}\left(y^{*}\right) \leq r .
$$

Let $x_{1}^{*}:=-A^{*} y^{*}, x_{2}^{*}:=A^{*} y^{*}, r_{1}:=f_{\bar{u}_{1}}^{*}\left(-A^{*} y^{*}\right)$ and $r_{2}:=r-r_{1}$. Then $\left(x_{1}^{*}, r_{1}\right) \in \operatorname{epi} f_{\bar{u}_{1}}^{*},\left(\bar{y}^{*}, r_{2}\right) \in$ epi $g_{\bar{u}_{2}}^{*}$ and $\left(x_{2}^{*}, r_{2}\right) \in\left(A^{*} \times \operatorname{id}_{\mathbb{R}}\right)\left(\right.$ epi $\left.g_{\bar{u}_{2}}^{*}\right)$. Thus,

$$
\begin{aligned}
(0, r) & =\left(x_{1}^{*}, r_{1}\right)+\left(x_{2}^{*}, r_{2}\right) \\
& \in \operatorname{epi} f_{\bar{u}_{1}}^{*}+\left(A^{*} \times \operatorname{id}_{\mathbb{R}}\right)\left(\operatorname{epi} g_{\bar{u}_{2}}^{*}\right) \\
& \in \bigcup_{u \in U}\left(\operatorname{epi} f_{u_{1}}^{*}+\left(A^{*} \times \operatorname{id} \mathbb{R}_{\mathbb{R}}\right)\left(\text { epi } g_{u_{2}}^{*}\right)\right) .
\end{aligned}
$$

(ii) Define the function $h: X \rightarrow \overline{\mathbb{R}}$ by

$$
h(x):=\sup _{u \in U}\left\{f_{u_{1}}(x)+g_{u_{2}}(A x)\right\} \quad \text { for each } x \in X .
$$

Then, by definition,

$$
h^{*}(0)=\sup _{x \in X}\left\{-\sup _{u \in U}\left\{f_{u_{1}}(x)+g_{u_{2}}(A x)\right\}\right\}=-\inf _{x \in X} \sup _{u \in U}\left\{f_{u_{1}}(x)+g_{u_{2}}(A x)\right\}=-v(\tilde{P}) .
$$

This means

$$
v(\tilde{P}) \geq-r \quad \Leftrightarrow \quad h^{*}(0) \leq r \quad \Leftrightarrow \quad(0, r) \in \operatorname{epi} h^{*} .
$$

Note by [22], Proposition 4.1, that

$$
\operatorname{epi} h^{*}=\operatorname{cl}\left[\operatorname{co} \bigcup_{u \in U} \operatorname{epi}\left(f_{u_{1}}+g_{u_{2}} \circ A\right)^{*}\right] \text {. }
$$

It follows from (3.17) that

$$
v(\tilde{P}) \geq-r \quad \Leftrightarrow \quad(0, r) \in \operatorname{cl}\left[\operatorname{co} \bigcup_{u \in U} \operatorname{epi}\left(f_{u_{1}}+g_{u_{2}} \circ A\right)^{*}\right] .
$$

The proof is complete.

The following theorem provides a characterization for the strong duality between $(P)$ and $(D)$ in terms of the $(A F R C)$ and the $(F R C)$.

Theorem 3.1 Consider the following statements:

(i) The family $\left(f_{u_{1}}, g_{u_{2}} ; A ; U\right)$ satisfies the (AFRC).

(ii) The strong duality holds between $(P)$ and $(D)$.

(iii) The family $\left(f_{u_{1}}, g_{u_{2}} ; A ; U\right)$ satisfies the (FRC).

Then (i) $\Rightarrow$ (ii) $\Rightarrow$ (iii). Furthermore, (i) $\Leftrightarrow$ (ii) $\Leftrightarrow$ (iii) if and only if

$$
\bigcup_{u \in U} \operatorname{epi}\left(f_{u_{1}}+g_{u_{2}} \circ A\right)^{*} \cap(\{0\} \times \mathbb{R}) \quad \text { is } w^{*} \text {-closed } .
$$


Proof (i) $\Rightarrow$ (ii) Suppose that the family $\left(f_{u_{1}}, g_{u_{2}} ; A ; U\right)$ satisfies the $(A F R C)$. If $v(P)=-\infty$, then, by the weak duality, $v(D)=-\infty$. Hence, the conclusion follows automatically. Below we assume that $-r:=v(P) \in \mathbb{R}$. Then, by (3.6), $r=\inf _{u \in U}\left(f_{u_{1}}+g_{u_{2}} \circ A\right)^{*}(0)$. Thus, for each $\varepsilon>0$, there exists $\bar{u}=\left(\bar{u}_{1}, \bar{u}_{2}\right) \in U$ such that

$$
\left(f_{\bar{u}_{1}}+g_{\bar{u}_{2}} \circ A\right)^{*}(0) \leq r+\varepsilon .
$$

This implies that

$$
(0, r+\varepsilon) \in \operatorname{epi}\left(f_{\bar{u}_{1}}+g_{\bar{u}_{2}} \circ A\right)^{*} \subseteq \bigcup_{u \in U} \operatorname{epi}\left(f_{u_{1}}+g_{u_{2}} \circ A\right)^{*} .
$$

Letting $\varepsilon \rightarrow 0$. We get that

$$
(0, r) \in \operatorname{cl}\left[\bigcup_{u \in U} \operatorname{epi}\left(f_{u_{1}}+g_{u_{2}} \circ A\right)^{*}\right] \subseteq \bigcup_{u \in U}\left(\operatorname{epi} f_{u_{1}}^{*}+\left(A^{*} \times \operatorname{id} \mathbb{R}\right)\left(\operatorname{epi} g_{u_{2}}^{*}\right)\right)
$$

where the last conclusion holds by the $(A F R C)$. Then, by Lemma 3.1(i), there exist $\bar{u}=$ $\left(\bar{u}_{1}, \bar{u}_{2}\right) \in U$ and $y^{*} \in Y^{*}$ such that (3.16) holds. Thus, by definition of $v(D)$,

$$
v(D) \geq-g_{\bar{u}_{2}}^{*}\left(y^{*}\right)-f_{\bar{u}_{1}}^{*}\left(-A^{*} y^{*}\right) \geq-r=v(P) .
$$

Therefore, by the weak duality, we see that $v(P)=v(D)$ and $\bar{u}=\left(\bar{u}_{1}, \bar{u}_{2}\right) \in U$ and $y^{*} \in Y^{*}$ are the optimal solution of $(D)$.

(ii) $\Rightarrow$ (iii) Suppose that the strong duality holds between $(P)$ and $(D)$. Let $(0, r) \in$ $\bigcup_{u \in U} \operatorname{epi}\left(f_{u_{1}}+g_{u_{2}} \circ A\right)^{*}$. Then there exists $\hat{u}=\left(\hat{u}_{1}, \hat{u}_{2}\right) \in U$ such that $(0, r) \in \operatorname{epi}\left(f_{\hat{u}_{1}}+g_{\hat{u}_{2}} \circ\right.$ $A)^{*}$. Thus,

$$
-r \leq-\left(f_{\hat{u}_{1}}+g_{\hat{u}_{2}} \circ A\right)^{*}(0) \leq-\inf _{u \in U}\left(f_{u_{1}}+g_{u_{2}} \circ A\right)^{*}(0)=v(P),
$$

where the last equality holds by (3.6). Hence, by (ii), we see that there exist $\bar{u}=\left(\bar{u}_{1}, \bar{u}_{2}\right) \in U$ and $y^{*} \in Y^{*}$ such that (3.16) holds. This together with Lemma 3.1(i) implies that $(0, r) \in$ $\bigcup_{u \in U}\left(\right.$ epi $f_{u_{1}}^{*}+\left(A^{*} \times \operatorname{id}_{\mathbb{R}}\right)\left(\right.$ epi $\left.\left.g_{u_{2}}^{*}\right)\right)$. Therefore, (3.9) is seen to hold and the implication (ii) $\Rightarrow$ (iii) is proved.

Suppose that (3.18) holds. Then the $(F R C)$ is equivalent to the $(A F R C)$. Thus, (i) $\Leftrightarrow$ (ii) $\Leftrightarrow$ (iii) and the proof is complete.

By Theorem 3.1 and Proposition 3.1, we get the following theorem straightforwardly.

\section{Theorem 3.2 Consider the following statements.}

(i) The family $\left(f_{u_{1}}, g_{u_{2}} ; A ; U\right)$ satisfies the $(A C C)$.

(ii) The stable strong duality holds between $(P)$ and $(D)$.

(iii) The family $\left(f_{u_{1}}, g_{u_{2}} ; A ; U\right)$ satisfies the (CC).

Then (i) $\Rightarrow$ (ii) $\Rightarrow$ (iii). Furthermore, (i) $\Leftrightarrow$ (ii) $\Leftrightarrow$ (iii) if and only if

$$
\bigcup_{u \in U} \operatorname{epi}\left(f_{u_{1}}+g_{u_{2}} \circ A\right)^{*} \quad \text { is } w^{*} \text {-closed }
$$


The following theorem provides a characterization for the strong duality and the stable strong duality to hold.

Theorem 3.3 Suppose that (3.14) holds. Then the following assertions hold.

(i) The strong duality holds between $(\tilde{P})$ and $(D)$ if and only if the family $\left(f_{u_{1}}, g_{u_{2}} ; A ; U\right)$ satisfies the (SFRC);

(ii) The stable strong duality holds between $(\tilde{P})$ and $(D)$ if and only if the family $\left(f_{u_{1}}, g_{u_{2}} ; A\right)$ satisfies the $(S C C)$.

Proof Assertion (ii) is a global version of assertion (i). Hence, by Proposition 3.1, we only need to prove (i). To do this, suppose that the strong duality holds between $(\tilde{P})$ and $(D)$. Let $(0, r) \in \operatorname{cl}\left[\operatorname{co} \bigcup_{u \in U} \operatorname{epi}\left(f_{u_{1}}+g_{u_{2}} \circ A\right)^{*}\right]$. Then, by Lemma 3.1(ii), we have that $v(\tilde{P}) \geq-r$. Since the strong duality holds between $(\tilde{P})$ and $(D)$, it follows that $v(D) \geq-r$ and there exist $\bar{u}=\left(\bar{u}_{1}, \bar{u}_{2}\right) \in U$ and $y^{*} \in Y^{*}$ such that (3.16) holds. This together with Lemma 3.1(i) implies that $(0, r) \in \bigcup_{u \in U}\left(\right.$ epi $f_{u_{1}}^{*}+\left(A^{*} \times \mathrm{id}_{\mathbb{R}}\right)\left(\right.$ epi $\left.\left.g_{u_{2}}^{*}\right)\right)$. Therefore, (3.7) holds.

Conversely, suppose that the family $\left(f_{u_{1}}, g_{u_{2}} ; A ; U\right)$ satisfies the $(S F R C)$. If $v(\tilde{P})=-\infty$, then, by the weak duality, $v(D)=-\infty$. Hence, the conclusion follows automatically. Below we assume that $-r:=v(\tilde{P}) \in \mathbb{R}$. Then, by Lemma 3.1(ii),

$$
(0, r) \in \mathrm{cl}\left[\operatorname{co} \bigcup_{u \in U} \operatorname{epi}\left(f_{u_{1}}+g_{u_{2}} \circ A\right)^{*}\right] \subseteq \bigcup_{u \in U}\left(\operatorname{epi} f_{u_{1}}^{*}+\left(A^{*} \times \operatorname{id} \mathbb{R}_{\mathbb{R}}\right)\left(\operatorname{epi} g_{u_{2}}^{*}\right)\right),
$$

where the last conclusion holds because of the (SFRC). This together with Lemma 3.1(i) implies that there exist $\bar{u}=\left(\bar{u}_{1}, \bar{u}_{2}\right) \in U$ and $y^{*} \in Y^{*}$ such that (3.16) holds. It follows that

$$
v(D) \geq-g_{\bar{u}_{2}}^{*}\left(y^{*}\right)-f_{\bar{u}_{1}}^{*}\left(-A^{*} y^{*}\right) \geq-r=v(\tilde{P}) .
$$

Thus, by the weak duality, we see that $v(\tilde{P})=v(D)$ and $\bar{u} \in U, y^{*} \in Y^{*}$ are the optimal solution of $(D)$. Therefore, the strong duality holds between $(\tilde{P})$ and $(D)$ and the proof is complete.

Remark 3.3 Let $X, Y, Z$ be Banach spaces and let $U_{1}, U_{2}$ be subsets of $Z$. Li et al. established in [16], Theorem 5.1, the strong duality between $(\tilde{P})$ and $(D)$ under the assumptions that (3.14) holds and

$$
\bigcup_{u \in U}\left(\text { epi } f_{u_{1}}^{*}+\left(A^{*} \times \operatorname{id} \mathbb{R}_{\mathbb{R}}\right)\left(\text { epi } g_{u_{2}}^{*}\right)\right) \quad \text { is } w^{*} \text {-closed and convex. }
$$

In this case, it is easy to see that (3.19) is equivalent to the (SFRC). Thus, [16], Theorem 5.1, follows from Theorem 3.3 directly.

The corollary follows directly from Theorem 3.3, Theorem 3.1 and Definition 3.2. It provides the friendships between the strong duality of $(\tilde{P})$ and $(D)$ and the strong duality of $(P)$ and $(D)$.

Corollary 3.1 Suppose that (3.14) holds. Then the following statements are equivalent.

(i) The strong duality holds between $(\tilde{P})$ and $(D)$. 
(ii) The strong duality holds between $(P)$ and $(D)$, and $\bigcup_{u \in U} \operatorname{epi}\left(f_{u_{1}}+g_{u_{2}} \circ A\right)^{*} \cap(\{0\} \times \mathbb{R})$ is $w^{*}$-closed and convex.

Combining Corollary 3.1 and Proposition 3.1, we have the following corollary.

Corollary 3.2 Suppose that (3.14) holds. Then the following statements are equivalent.

(i) The stable strong duality holds between $(\tilde{P})$ and $(D)$.

(ii) The stable strong duality holds between $(P)$ and $(D)$, and $\bigcup_{u \in U}$ epi $\left(f_{u_{1}}+g_{u_{2}} \circ A\right)^{*}$ is $w^{*}$-closed and convex.

\section{Total duality}

Recall that the problem $\left(P_{p}\right)$ and the corresponding dual problem $\left(D_{p}\right)$ are defined by (3.1) and (3.2), respectively. Let $p \in X^{*}$ and $u=\left(u_{1}, u_{2}\right) \in U$, we define the subproblem of the problem $\left(P_{p}\right)$ by

$$
\left(P_{p}^{u}\right) \quad \inf _{x \in X}\left\{f_{u_{1}}(x)+g_{u_{2}}(A x)-\langle p, x\rangle\right\}
$$

and use $v\left(P_{p}^{u}\right)$ to denote the optimal value of the problem $\left(P_{p}^{u}\right)$. Let $\left(u_{1}, u_{2}\right) \in U$ and let $x_{0} \in \bigcap_{u \in U}\left[\left(\operatorname{dom} f_{u_{1}}\right) \cap A^{-1}\left(\operatorname{dom} g_{u_{2}}\right)\right]$. We say the Moreau-Rockafellar formula holds at $\left(u_{1}, u_{2} ; x_{0}\right)$ if

$$
\partial\left(f_{u_{1}}+g_{u_{2}} \circ A\right)\left(x_{0}\right)=\partial f_{u_{1}}\left(x_{0}\right)+A^{*} \partial g_{u_{2}}\left(A x_{0}\right)
$$

This section is devoted to the study of characterizing the total dualities. For this purpose, let $p \in X^{*}$ and $S\left(P_{p}\right)$ denote the optimal solution set of $\left(P_{p}\right)$, that is,

$$
\left(\bar{u}_{1}, \bar{u}_{2} ; x_{0}\right) \in S\left(P_{p}\right) \quad \Leftrightarrow \quad f_{\bar{u}_{1}}\left(x_{0}\right)+g_{\bar{u}_{2}}\left(A x_{0}\right)-\left\langle p, x_{0}\right\rangle=v\left(P_{p}\right),
$$

and for each $u=\left(u_{1}, u_{2}\right) \in U$, let $S\left(P_{p}^{u}\right)$ denote the optimal solution set of $\left(P_{p}^{u}\right)$, that is,

$$
x_{0} \in S\left(P_{p}^{u}\right) \quad \Leftrightarrow \quad f_{u_{1}}\left(x_{0}\right)+g_{u_{2}}\left(A x_{0}\right)-\left\langle p, x_{0}\right\rangle=v\left(P_{p}^{u}\right)
$$

Theorem 4.1 Let $x_{0} \in \bigcap_{u \in U}\left[\left(\operatorname{dom} f_{u_{1}}\right) \cap A^{-1}\left(\operatorname{dom} g_{u_{2}}\right)\right]$ and $\bar{u}=\left(\bar{u}_{1}, \bar{u}_{2}\right) \in U$. If the MoreauRockafellarformula holds at $\left(\bar{u}_{1}, \bar{u}_{2} ; x_{0}\right)$, then, for each $p \in X^{*}$ satisfying $\left(\bar{u}_{1}, \bar{u}_{2} ; x_{0}\right) \in S\left(P_{p}\right)$,

$$
v\left(P_{p}\right)=\max _{y^{*} \in Y^{*}}\left\{-f_{\bar{u}_{1}}^{*}\left(p-A^{*} y^{*}\right)-g_{\bar{u}_{2}}^{*}\left(y^{*}\right)\right\}=v\left(D_{p}\right) .
$$

Conversely, if (4.2) holdsfor each $p \in X^{*}$ satisfying $x_{0} \in S\left(P_{p}^{\bar{u}}\right)$, then the Moreau-Rockafellar formula holds at $\left(\bar{u}_{1}, \bar{u}_{2} ; x_{0}\right)$.

Proof Suppose that the Moreau-Rockafellar formula holds at $\left(\bar{u}_{1}, \bar{u}_{2} ; x_{0}\right)$. Let $p \in X^{*}$ be such that $\left(\bar{u}_{1}, \bar{u}_{2} ; x_{0}\right) \in S\left(P_{p}\right)$, that is,

$$
f_{\bar{u}_{1}}\left(x_{0}\right)+g_{\bar{u}_{2}}\left(A x_{0}\right)-\left\langle p, x_{0}\right\rangle=v\left(P_{p}\right) .
$$


Then, by (2.3), we see that $0 \in \partial\left(f_{\bar{u}_{1}}-p+g_{\bar{u}_{2}} \circ A\right)\left(x_{0}\right)$, which is equivalent to $p \in \partial\left(f_{\bar{u}_{1}}+g_{\bar{u}_{2}} \circ\right.$ $A)\left(x_{0}\right)$. This together with (4.1) implies that $p \in \partial f_{\bar{u}_{1}}\left(x_{0}\right)+A^{*} \partial g_{\bar{u}_{2}}\left(A x_{0}\right)$. Therefore, there exist $p_{1} \in \partial f_{\bar{u}_{1}}\left(x_{0}\right)$ and $p_{2} \in \partial g_{\bar{u}_{2}}\left(A x_{0}\right)$ such that

$$
p=p_{1}+A^{*} p_{2}
$$

Applying the Young equality (2.4), one has

$$
f_{\bar{u}_{1}}^{*}\left(p_{1}\right)+f_{\bar{u}_{1}}\left(x_{0}\right)=\left\langle p_{1}, x_{0}\right\rangle \quad \text { and } \quad g_{\bar{u}_{2}}^{*}\left(p_{2}\right)+g_{\bar{u}_{2}}\left(A x_{0}\right)=\left\langle p_{2}, A x_{0}\right\rangle .
$$

Noting that $\left\langle p_{2}, A x_{0}\right\rangle=\left\langle A^{*} p_{2}, x_{0}\right\rangle$ and using (4.4),

$$
f_{\bar{u}_{1}}^{*}\left(p_{1}\right)+g_{\bar{u}_{2}}^{*}\left(p_{2}\right)+f_{\bar{u}_{1}}\left(x_{0}\right)+g_{\bar{u}_{2}}\left(A x_{0}\right)=\left\langle p_{1}, x_{0}\right\rangle+\left\langle p_{2}, A x_{0}\right\rangle=\left\langle p, x_{0}\right\rangle .
$$

Hence,

$$
-f_{\bar{u}_{1}}^{*}\left(p_{1}\right)-g_{\bar{u}_{2}}^{*}\left(p_{2}\right)=f_{\bar{u}_{1}}\left(x_{0}\right)+g_{\bar{u}_{2}}\left(A x_{0}\right)-\left\langle p, x_{0}\right\rangle=v\left(P_{p}\right)
$$

while, by the definition of $v\left(D_{p}\right)$ and noting $p_{2} \in Y^{*}$, one has that

$$
v\left(D_{p}\right) \geq-f_{\bar{u}_{1}}^{*}\left(p-A^{*} p_{2}\right)-g_{\bar{u}_{2}}^{*}\left(p_{2}\right)=-f_{\bar{u}_{1}}^{*}\left(p_{1}\right)-g_{\bar{u}_{2}}^{*}\left(p_{2}\right)=v\left(P_{p}\right) .
$$

Combining this with the weak duality, we see that

$$
v\left(D_{p}\right)=-f_{\bar{u}_{1}}^{*}\left(p-A^{*} p_{2}\right)-g_{\bar{u}_{2}}^{*}\left(p_{2}\right)=v\left(P_{p}\right) .
$$

Thus (4.2) holds.

Conversely, let $p \in \partial\left(f_{\bar{u}_{1}}+g_{\bar{u}_{2}} \circ A\right)\left(x_{0}\right)$. Then $0 \in \partial\left(f_{\bar{u}_{1}}+g_{\bar{u}_{2}} \circ A-p\right)\left(x_{0}\right)$ and by (2.3),

$$
f_{\bar{u}_{1}}\left(x_{0}\right)+g_{\bar{u}_{2}}\left(A x_{0}\right)-\left\langle p, x_{0}\right\rangle \leq f_{\bar{u}_{1}}(x)+g_{\bar{u}_{2}}(A x)-\langle p, x\rangle \quad \text { for each } x \in X .
$$

This means $x_{0} \in S\left(P_{p}^{\bar{u}}\right)$. Thus, by (4.2), we have that

$$
f_{\bar{u}_{1}}\left(x_{0}\right)+g_{\bar{u}_{2}}\left(A x_{0}\right)-\left\langle p, x_{0}\right\rangle \leq \max _{y^{*} \in Y^{*}}\left\{-f_{\bar{u}_{1}}^{*}\left(p-A^{*} y^{*}\right)-g_{\bar{u}_{2}}^{*}\left(y^{*}\right)\right\} .
$$

This implies that there exists $q \in Y^{*}$ such that

$$
f_{\bar{u}_{1}}\left(x_{0}\right)+g_{\bar{u}_{2}}\left(A x_{0}\right)-\left\langle p, x_{0}\right\rangle \leq-f_{\bar{u}_{1}}^{*}\left(p-A^{*} q\right)-g_{\bar{u}_{2}}^{*}(q) .
$$

Noting the above inequality and using the definition of the conjugate function, we see that

$$
0 \leq g_{\bar{u}_{2}}^{*}(q)+g_{\bar{u}_{2}}\left(A x_{0}\right)-\left\langle q, A x_{0}\right\rangle \leq\left\langle p-A^{*} q, x_{0}\right\rangle-f_{\bar{u}_{1}}\left(x_{0}\right)-f_{\bar{u}_{1}}^{*}\left(p-A^{*} q\right) \leq 0 .
$$

It follows that

$$
g_{\bar{u}_{2}}^{*}(q)+g_{\bar{u}_{2}}\left(A x_{0}\right)-\left\langle q, A x_{0}\right\rangle=0 \text { and }\left\langle p-A^{*} q, x_{0}\right\rangle-f_{\bar{u}_{1}}\left(x_{0}\right)-f_{\bar{u}_{1}}^{*}\left(p-A^{*} q\right)=0 .
$$


Combing this with (2.4), we have that $q \in \partial g_{\bar{u}_{2}}\left(x_{0}\right)$ and $p-A^{*} q \in \partial f_{\bar{u}_{1}}\left(x_{0}\right)$. Consequently,

$$
p=p-A^{*} q+A^{*} q \in \partial f_{\bar{u}_{1}}\left(x_{0}\right)+A^{*} \partial g_{\bar{u}_{2}}\left(x_{0}\right)
$$

and the set on the left-hand side of the Moreau-Rockafellar formula is contained in the set on the right-hand side. This completes the proof because the converse inclusion holds automatically.

\section{Remark 4.1}

(i) In the case when $U_{1}$ and $U_{2}$ are singletons, the Moreau-Rockafellar formula (4.1) reduces to the Moreau-Rockafellar formula in [23], p.47, which was also introduced in [2] to establish the stable total Fenchel duality for $(\mathcal{P})$ and $(\mathcal{D})$ (see (1.1) and (1.2)).

(ii) By Theorem 4.1 and Theorem 3.2, it is easy to see that if (ACC) holds, then the Moreau-Rockafellar formula (4.1) holds at each $\left(u_{1}, u_{2} ; x_{0}\right) \in U_{1} \times U_{2} \times \bigcap_{u \in U}\left[\left(\operatorname{dom} f_{u_{1}}\right) \cap A^{-1}\left(\operatorname{dom} g_{u_{2}}\right)\right]$.

\section{Converse duality}

For each $p \in X$ and $q \in Y$, we consider the following perturbed robust optimization problem:

$$
\left(C P_{(p, q)}\right) \quad \sup _{u \in U} \inf _{x \in X}\left\{f_{u_{1}}(x-p)+g_{u_{2}}(A x+q)\right\}
$$

and the corresponding dual problem

$$
\left(C D_{(p, q)}\right) \quad \sup _{u \in U} \sup _{y^{*} \in Y^{*}}\left\{\left\langle A p+q, y^{*}\right\rangle-g_{u_{2}}^{*}\left(y^{*}\right)-f_{u_{1}}^{*}\left(-A^{*} y^{*}\right)\right\} .
$$

Let $u=\left(u_{1}, u_{2}\right) \in U$. We define the subproblem of $\left(C P_{(p, q)}\right)$ by

$$
\left(C P_{(p, q)}^{u}\right)=\inf _{x \in X}\left\{f_{u_{1}}(x-p)+g_{u_{2}}(A x+q)\right\}
$$

As before, we use $v\left(C P_{(p, q)}\right), v\left(C D_{(p, q)}\right)$ and $v\left(C P_{(p, q)}^{u}\right)$ to stand for the optimal values of the problems $\left(C P_{(p, q)}\right),\left(C D_{(p, q)}\right)$ and $\left(C P_{(p, q)}^{u}\right)$, respectively. Clearly, the weak duality holds, that is,

$$
\nu\left(C D_{(p, q)}\right) \leq v\left(C P_{(p, q)}\right) \quad \text { for each } p \in X \text { and } q \in Y \text {. }
$$

Moreover, by definitions, we have the following equalities:

$$
\begin{aligned}
& v\left(C P_{(p, q)}^{u}\right)=\left(g_{u_{2}} \square(-A) f_{u_{1}}\right)(A p+q), \\
& v\left(C P_{(p, q)}\right)=\sup _{u \in U}\left(g_{u_{2}} \square(-A) f_{u_{1}}\right)(A p+q),
\end{aligned}
$$

and

$$
v\left(C D_{(p, q)}\right)=\sup _{u \in U}\left(g_{u_{2}}^{*}+f_{u_{1}}^{*} \circ\left(-A^{*}\right)\right)^{*}(A p+q) .
$$


In fact, (5.4) comes from the definitions, (5.3) follows from (5.2), and (5.2) holds because

$$
\begin{aligned}
\left(g_{u_{2}} \square(-A) f_{u_{1}}\right)(A p+q) & =\inf _{y \in Y}\left\{g_{u_{2}}(q-y)+(-A) f_{u_{1}}(A p+y)\right\} \\
& =\inf _{y \in Y}\left\{g_{u_{2}}(q-y)+\inf \left\{f_{u_{1}}(x-p):-A(x-p)=A p+y, x \in X\right\}\right\} \\
& =\inf _{x \in X}\left\{g_{u_{2}}(q+A x)+f_{u_{1}}(x-p)\right\} \\
& =v\left(C P_{(p, q)}^{u}\right) .
\end{aligned}
$$

In particular, in the case when $p=q=0$, problems $\left(C P_{(p, q)}\right)$ and $\left(C D_{(p, q)}\right)$ are just as the problem $(P)$ and problem $(D)$, respectively. Thus, by (5.3), (5.4) and (5.2), we see that

$$
\begin{aligned}
& v\left(P^{u}\right)=\left(g_{u_{2}} \square(-A) f_{u_{1}}\right)(0), \\
& v(P)=\sup _{u \in U}\left(g_{u_{2}} \square(-A) f_{u_{1}}\right)(0),
\end{aligned}
$$

and

$$
v(D)=\sup _{u \in U}\left(g_{u_{2}}^{*}+f_{u_{1}}^{*} \circ\left(-A^{*}\right)\right)^{*}(0)
$$

Definition 5.1 It is said that

(i) the converse duality holds between $(P)$ and $(D)$ if $v(P)=v(D)$ and for each $u \in U$ satisfying $v\left(P^{u}\right)=v(P)$, the problem $\left(P^{u}\right)$ has an optimal solution;

(ii) the stable converse duality holds between $(P)$ and $(D)$ if the converse duality holds between $\left(C P_{(p, q)}\right)$ and $\left(C D_{(p, q)}\right)$ for each $p \in X$ and $q \in Y$.

To study the converse duality and the stable converse duality between $(P)$ and $(D)$, we introduce the following regularity conditions.

Definition 5.2 The family $\left(f_{u_{1}}, g_{u_{2}} ; A ; U\right)$ is said to satisfy

(i) the converse further regularity condition $(C F R C)$ if

$$
\begin{aligned}
& \bigcap_{u \in U} \operatorname{epi}\left(g_{u_{2}}^{*}+f_{u_{1}}^{*} \circ\left(-A^{*}\right)\right)^{*} \cap(\{0\} \times \mathbb{R}) \\
& \subseteq \bigcap_{u \in U}\left[\left(\operatorname{epi} g_{u_{2}}+((-A) \times \operatorname{id} \mathbb{R})\left(\operatorname{epi} f_{u_{1}}\right)\right] \cap(\{0\} \times \mathbb{R})\right.
\end{aligned}
$$

(ii) the converse closure condition $(C C C)$ if

$$
\bigcap_{u \in U} \operatorname{epi}\left(g_{u_{2}}^{*}+f_{u_{1}}^{*} \circ\left(-A^{*}\right)\right)^{*} \subseteq \bigcap_{u \in U}\left[\left(\operatorname{epi} g_{u_{2}}+\left((-A) \times \operatorname{id}_{\mathbb{R}}\right)\left(\operatorname{epi} f_{u_{1}}\right)\right]\right.
$$

Remark 5.1 Recall from [2], Definition 6.2, that the triple $(f, g ; A)$ satisfies the converse $(F R C)_{A}$ if

$$
\left(g^{*}+f^{*} \circ\left(-A^{*}\right)\right)^{*}(0) \geq(g \square(-A) f)(0),
$$


and there exists $y \in Y$ such that $(g \square(-A) f)(0)=g(-y)+(-A) f(y)$ and the infimum in the definition of $(-A) f(y)$ is attained, where $g \square(-A) f$ denotes the infimal convolution of $g$ and $(-A) f$, and from [2], Definition 6.5, that the triple $(f, g ; A)$ satisfies the converse $(C C)_{A}$ if

$$
\operatorname{epi}\left(g^{*}+f^{*} \circ\left(-A^{*}\right)\right)^{*}=\operatorname{epi} g+\left((-A) \times \mathrm{id}_{\mathbb{R}}\right)(\text { epi } f) .
$$

By [2], Proposition 6.3, the converse $(F R C)_{A}$ for the triple $(f, g ; A)$ is equivalent to

$$
\operatorname{epi}\left(g^{*}+f^{*} \circ\left(-A^{*}\right)\right)^{*} \cap(\{0\} \times \mathbb{R}) \subseteq\left(\text { epi } g+\left((-A) \times \operatorname{id}_{\mathbb{R}}\right)(\text { epi } f)\right) \cap(\{0\} \times \mathbb{R}) .
$$

Thus, in the case when $U_{1}$ and $U_{2}$ are singletons, the (CFRC) and (CCC) for the family $\left(f_{u_{1}}, g_{u_{2}} ; A ; U\right)$ are reduced into the converse $(F R C)_{A}$ and the converse $(C C)_{A}$, respectively, for the triple $(f, g ; A)$.

\section{Theorem 5.1}

(i) The family $\left(f_{u_{1}}, g_{u_{2}} ; A ; U\right)$ satisfies the $(C F R C)$ if and only if the converse duality holds between $(P)$ and $(D)$.

(ii) The family $\left(f_{u_{1}}, g_{u_{2}} ; A ; U\right)$ satisfies the $(C C C)$ if and only if the stable converse duality holds between $(P)$ and $(D)$.

Proof The proof of (i) is similar to that of (ii). Hence, we only need to prove (ii). To do it, suppose that the family $\left(f_{u_{1}}, g_{u_{2}} ; A ; U\right)$ satisfies the (CCC). Let $p \in X$ and $q \in Y$ and let $r:=v\left(C D_{(p, q)}\right)$. Then, by (5.4), $r=\sup _{u \in U}\left(g_{u_{2}}^{*}+f_{u_{1}}^{*} \circ\left(-A^{*}\right)\right)^{*}(A p+q)$. Thus, for each $u \in U$,

$$
r \geq\left(g_{u_{2}}^{*}+f_{u_{1}}^{*} \circ\left(-A^{*}\right)\right)^{*}(A p+q)
$$

This implies that $(A p+q, r) \in \operatorname{epi}\left(g_{u_{2}}^{*}+f_{u_{1}}^{*} \circ\left(-A^{*}\right)\right)^{*}$, and by the arbitrariness of $u \in U$,

$$
(A p+q, r) \in \bigcap_{u \in U} \operatorname{epi}\left(g_{u_{2}}^{*}+f_{u_{1}}^{*} \circ\left(-A^{*}\right)\right)^{*} \subseteq \bigcap_{u \in U}\left[\left(\operatorname{epi} g_{u_{2}}+\left((-A) \times \operatorname{id} \mathbb{R}_{\mathbb{R}}\right)\left(\operatorname{epi} f_{u_{1}}\right)\right]\right.
$$

thanks to the $(C C C)$. Let $u \in U$ be arbitrary. Then

$$
(A p+q, r) \in \operatorname{epi} g_{u_{2}}+\left((-A) \times \operatorname{id}_{\mathbb{R}}\right)\left(\operatorname{epi} f_{u_{1}}\right) .
$$

Thus, there exist $\left(y_{1}, r_{1}\right) \in \operatorname{epi} g_{u_{2}}$ and $\left(y_{2}, r_{2}\right) \in((-A) \times \operatorname{id} \mathbb{R})\left(\operatorname{epi} f_{u_{1}}\right)$ such that

$$
y_{1}+y_{2}=A p+q \text { and } r_{1}+r_{2}=r .
$$

Moreover, there exists $\hat{x} \in X$ such that $-A \hat{x}=y_{2}$ and $\left(\hat{x}, r_{2}\right) \in \operatorname{epi} f_{u_{1}}$. Consequently, $f_{u_{1}}(\hat{x}) \leq$ $r_{2}$ and $g_{u_{2}}\left(y_{1}\right) \leq r_{1}$. Therefore,

$$
\left(g_{u_{2}} \square(-A) f_{u_{1}}\right)(A p+q) \leq g_{u_{2}}(A p+q+A \hat{x})+f_{u_{1}}(\hat{x})=g_{u_{2}}\left(y_{1}\right)+f_{u_{1}}(\hat{x}) \leq r_{1}+r_{2}=r .
$$

Note that $u$ is arbitrary, it follows that

$$
\sup _{u \in U}\left(g_{u_{2}} \square(-A) f_{u_{1}}\right)(A p+q) \leq r .
$$


This together with (5.3) implies that $v\left(C P_{(p, q)}\right) \leq r$. Therefore, by the weak duality, $v\left(C P_{(p, q)}\right)=v\left(C D_{(p, q)}\right)$ and $\hat{x}+p$ is the optimal solution of $\left(C P_{(p, q)}^{u}\right)$ which satisfies $v\left(C P_{(p, q)}^{u}\right)=$ $\nu\left(C P_{(p, q)}\right)$.

Conversely, suppose that the stable converse duality holds between $(P)$ and $(D)$. Let $p \in$ $X, q \in Y$ and let $\bar{u}=\left(\bar{u}_{1}, \bar{u}_{2}\right) \in \bar{U}$ be such that $v\left(C P_{(p, q)}\right)=v\left(C P_{(p, q)}^{\bar{u}}\right)$. Then there exists $x_{0} \in X$ such that

$$
v\left(C P_{(p, q)}^{\bar{u}}\right)=f_{\bar{u}_{1}}\left(x_{0}-p\right)+g_{\bar{u}_{2}}\left(A x_{0}+q\right)=v\left(C P_{(p, q)}\right) .
$$

Let $(A p+q, r) \in \bigcap_{u \in U}$ epi $\left(g_{u_{2}}^{*}+f_{u_{1}}^{*} \circ\left(-A^{*}\right)\right)^{*}$. Then, for each $u \in U$,

$$
r \geq\left(g_{u_{2}}^{*}+f_{u_{1}}^{*} \circ\left(-A^{*}\right)\right)^{*}(A p+q) .
$$

This together with (5.4) implies that

$$
r \geq \sup _{u \in U}\left(g_{u_{2}}^{*}+f_{u_{1}}^{*} \circ\left(-A^{*}\right)\right)^{*}(A p+q)=v\left(C D_{(p, q)}\right)=v\left(C P_{(p, q)}\right)
$$

and

$$
r \geq f_{\bar{u}_{1}}\left(x_{0}-p\right)+g_{\bar{u}_{2}}\left(A x_{0}+q\right)=\sup _{u \in U}\left\{f_{u_{1}}\left(x_{0}-p\right)+g_{u_{2}}\left(A x_{0}+q\right)\right\},
$$

by (5.9) and the definitions of $\left(C P_{(p, q)}\right)$ and $\left(C D_{(p, q)}\right)$. Thus,

$$
r \geq f_{u_{1}}\left(x_{0}-p\right)+g_{u_{2}}\left(A x_{0}+q\right) \quad \text { for each } u \in U
$$

This means, for each $u=\left(u_{1}, u_{2}\right) \in U$,

$$
\left(-A x_{0}+A p, r-g_{u_{2}}\left(A x_{0}+q\right)\right) \in\left((-A) \times \mathrm{id}_{\mathbb{R}}\right)\left(\text { epi } f_{u_{1}}\right) .
$$

Note that $\left(A x_{0}+q, g_{u_{2}}\left(A x_{0}+q\right)\right) \in$ epi $g_{u_{2}}$ holds for each $u_{2} \in U_{2}$. Then, for each $u \in U$,

$$
\begin{aligned}
(A p+q, r) & =\left(A x_{0}+q, g_{u_{2}}\left(A x_{0}+q\right)\right)+\left(-A x_{0}+A p, r-g_{u_{2}}\left(A x_{0}+q\right)\right) \\
& \in\left(\operatorname{epi} g_{u_{2}}+\left((-A) \times \operatorname{id}_{\mathbb{R}}\right)\left(\operatorname{epi} f_{u_{1}}\right)\right.
\end{aligned}
$$

and hence

$$
(A p+q, r) \in \bigcap_{u \in U}\left[\left(\operatorname{epi}_{u_{2}}+\left((-A) \times \operatorname{id}_{\mathbb{R}}\right)\left(\text { epi } f_{u_{1}}\right)\right] .\right.
$$

Therefore, by the arbitrariness of $p \in X$ and $q \in Y$, the (CCC) holds. The proof is complete.

In the remainder of this section, we assume that $f_{u_{1}}: X \rightarrow \overline{\mathbb{R}}, u_{1} \in U_{1}$ and $g_{u_{2}}: Y \rightarrow$ $\overline{\mathbb{R}}, u_{2} \in U_{2}$ are proper lsc convex functions. We now present a necessary and sufficient condition for the total converse duality to hold. To do this, we first introduce two converse Moreau-Rockafellar formulae. 
Definition 5.3 Let $y_{0}^{*} \in \bigcap_{u \in U}\left[\left(\operatorname{dom} g_{u_{2}}^{*}\right) \cap(-A)^{*}\left(\operatorname{dom} f_{u_{1}}^{*}\right)\right]$ and $\bar{u}=\left(\bar{u}_{1}, \bar{u}_{2}\right) \in U$. It is said that

(i) the strong converse Moreau-Rockafellar formula $(S C M R)$ holds at $\left(\bar{u}_{1}, \bar{u}_{2} ; y_{0}^{*}\right)$ if

$$
\partial\left(f_{\bar{u}_{1}}^{*} \circ\left(-A^{*}\right)+g_{\bar{u}_{2}}^{*}\right)\left(y_{0}^{*}\right) \subseteq \bigcap_{u \in U}\left[(-A) \partial f_{u_{1}}^{*}\left(-A^{*} y_{0}^{*}\right)+\partial g_{u_{2}}^{*}\left(y_{0}^{*}\right)\right]
$$

(ii) the converse Moreau-Rockafellar formula $(C M R)$ holds at $\left(\bar{u}_{1}, \bar{u}_{2} ; y_{0}^{*}\right)$ if

$$
\partial\left(f_{\bar{u}_{1}}^{*} \circ\left(-A^{*}\right)+g_{\bar{u}_{2}}^{*}\right)\left(y_{0}^{*}\right) \subseteq \bigcup_{u \in U}\left[(-A) \partial f_{u_{1}}^{*}\left(-A^{*} y_{0}^{*}\right)+\partial g_{u_{2}}^{*}\left(y_{0}^{*}\right)\right] .
$$

Remark 5.2 In the case when $U_{1}$ and $U_{2}$ are singletons, (5.10) and (5.11) are reduced to the converse Moreau-Rockafellar formula at $y_{0}^{*} \in Y^{*} \cap\left(\operatorname{dom} g^{*}\right) \cap(-A)^{*}\left(\operatorname{dom} f^{*}\right)$ (see [2], Definition 6.8), that is,

$$
\partial\left(f^{*} \circ\left(-A^{*}\right)+g^{*}\right)\left(y_{0}^{*}\right)=(-A) \partial f^{*}\left(-A^{*} y_{0}^{*}\right)+\partial g^{*}\left(y_{0}^{*}\right) .
$$

Let $u=\left(u_{1}, u_{2}\right) \in U$, we define the subproblem of the problem $\left(C D_{(p, q)}\right)$ by

$$
\left(C D_{(p, q)}^{u}\right)=\sup _{y \in Y^{*}}\left\{\left\langle A p+q, y^{*}\right\rangle-g_{u_{2}}^{*}\left(y^{*}\right)-f_{u_{1}}^{*}\left(-A^{*} y^{*}\right)\right\}
$$

and use $v\left(C D_{(p, q)}^{\bar{u}}\right)$ to denote the optimal value of the problem $\left(C D_{(p, q)}^{\bar{u}}\right)$. For each $p \in X$ and $q \in Y$, let $S(p, q)$ denote the solution set of the problem $\left(C D_{(p, q)}\right)$, that is,

$$
\left(\bar{u}_{1}, \bar{u}_{2} ; y_{0}^{*}\right) \in S(p, q) \Leftrightarrow\left\langle A p+q, y_{0}^{*}\right\rangle-g_{\bar{u}_{2}}^{*}\left(y_{0}^{*}\right)-f_{\bar{u}_{1}}^{*}\left(-A^{*} y_{0}^{*}\right)=v\left(C D_{(p, q)}\right),
$$

and let $S(p, q, u)$ denote the solution set of the problem $\left(C D_{(p, q)}^{u}\right)$, that is,

$$
y_{0}^{*} \in S(p, q, u) \Leftrightarrow\left\langle A p+q, y_{0}^{*}\right\rangle-g_{u_{2}}^{*}\left(y_{0}^{*}\right)-f_{u_{1}}^{*}\left(-A^{*} y_{0}^{*}\right)=v\left(C D_{(p, q)}^{u}\right) .
$$

Theorem 5.2 Let $y_{0}^{*} \in \bigcap_{u \in U}\left[\left(\operatorname{dom} g_{u_{2}}^{*}\right) \cap(-A)^{*}\left(\operatorname{dom} f_{u_{1}}^{*}\right)\right]$ and $\bar{u}=\left(\bar{u}_{1}, \bar{u}_{2}\right) \in U$.

(i) If the $(S C M R)$ holds at $\left(\bar{u}_{1}, \bar{u}_{2} ; y_{0}^{*}\right)$, then for each $p \in X$ and $q \in Y$ satisfying $\left(\bar{u}_{1}, \bar{u}_{2} ; y_{0}^{*}\right) \in S(p, q), v\left(C D_{(p, q)}\right)=v\left(C P_{(p, q)}\right)=v\left(C P_{(p, q)}^{\bar{u}}\right)$, and the problem $\left(C P_{(p, q)}^{\bar{u}}\right)$ has an optimal solution.

(ii) If for each $p \in X$ and $q \in Y$ satisfying $y_{0}^{*} \in S(p, q ; \bar{u}), v\left(C D_{(p, q)}^{\bar{u}}\right)=v\left(C P_{(p, q)}^{\bar{u}}\right)$, and the problem $\left(C P_{(p, q)}^{\bar{u}}\right)$ has an optimal solution, then the $(C M R)$ holds at $\left(\bar{u}_{1}, \bar{u}_{2} ; y_{0}^{*}\right)$.

Proof (i) Suppose that the (SCMR) holds at $\left(\bar{u}_{1}, \bar{u}_{2} ; y_{0}^{*}\right)$. Then (5.10) holds. Let $p \in X, q \in Y$ be such that $\left(\bar{u}_{1}, \bar{u}_{2} ; y_{0}^{*}\right) \in S(p, q)$. Then

$$
v\left(C D_{(p, q)}\right)=\left\langle A p+q, y_{0}^{*}\right\rangle-f_{\bar{u}_{1}}^{*}\left(-A^{*} y_{0}^{*}\right)-g_{\bar{u}_{2}}^{*}\left(y_{0}^{*}\right) .
$$

By (2.3), we see that $0 \in \partial\left(f_{\bar{u}_{1}}^{*} \circ\left(-A^{*}\right)+g_{\bar{u}_{2}}^{*}-(A p+q)\right)\left(y_{0}^{*}\right)$, which is equivalent to $A p+q \in$ $\partial\left(f_{\bar{u}_{1}}^{*} \circ\left(-A^{*}\right)+g_{\bar{u}_{2}}^{*}\right)\left(y_{0}^{*}\right)$. This together with (5.10) implies that

$$
(A p+q) \in \bigcap_{u \in U}\left[(-A) \partial f_{u_{1}}^{*}\left(-A^{*} y_{0}^{*}\right)+\partial g_{u_{2}}^{*}\left(y_{0}^{*}\right)\right] .
$$


Let $u=\left(u_{1}, u_{2}\right) \in U$. Then there exist $p_{1} \in \partial f_{u_{1}}^{*}\left(-A^{*} y_{0}^{*}\right)$ and $p_{2} \in \partial g_{u_{2}}^{*}\left(y_{0}^{*}\right)$ such that

$$
(-A) p_{1}+p_{2}=A p+q
$$

Applying the Young equality (2.4), one has

$$
f_{u_{1}}^{* *}\left(p_{1}\right)+f_{u_{1}}^{*}\left(-A^{*} y_{0}^{*}\right)=\left\langle p_{1}, A^{*} y_{0}^{*}\right\rangle \quad \text { and } \quad g_{u_{2}}^{* *}\left(p_{2}\right)+g_{u_{2}}^{*}\left(y_{0}^{*}\right)=\left\langle p_{2}, y_{0}^{*}\right\rangle
$$

Noting that $\left\langle p_{1}, A^{*} y_{0}^{*}\right\rangle=\left\langle A p_{1}, y_{0}^{*}\right\rangle$, it follows that

$$
f_{u_{1}}\left(p_{1}\right)+g_{u_{2}}\left(p_{2}\right)=f_{u_{1}}^{* *}\left(p_{1}\right)+g_{u_{2}}^{* *}\left(p_{2}\right)=\left\langle A p+q, y_{0}^{*}\right\rangle-f_{u_{1}}^{*}\left(-A^{*} y_{0}^{*}\right)-g_{u_{2}}^{*}\left(y_{0}^{*}\right)
$$

where the first equality holds because $f_{u_{1}}$ and $g_{u_{2}}$ are lsc functions. Then, by the arbitrariness of $u \in U$, we have that

$$
\sup _{u \in U}\left\{f_{u_{1}}\left(p_{1}\right)+g_{u_{2}}\left(p_{2}\right)\right\}=\sup _{u \in U}\left\{\left\langle A p+q, y_{0}^{*}\right\rangle-f_{u_{1}}^{*}\left(-A^{*} y_{0}^{*}\right)-g_{u_{2}}^{*}\left(y_{0}^{*}\right)\right\}=v\left(C D_{(p, q)}\right) .
$$

Moreover, by (5.14) and (5.16),

$$
f_{\bar{u}_{1}}\left(p_{1}\right)+g_{\bar{u}_{2}}\left(p_{2}\right)=\left\langle A p+q, y_{0}^{*}\right\rangle-f_{\bar{u}_{1}}^{*}\left(-A^{*} y_{0}^{*}\right)-g_{\bar{u}_{2}}^{*}\left(y_{0}^{*}\right)=v\left(C D_{(p, q)}\right)
$$

Thus,

$$
\sup _{u \in U}\left\{f_{u_{1}}\left(p_{1}\right)+g_{u_{2}}\left(p_{2}\right)\right\}=f_{\bar{u}_{1}}\left(p_{1}\right)+g_{\bar{u}_{2}}\left(p_{2}\right)=v\left(C D_{(p, q)}\right)
$$

while, by the definition of $\left(C P_{(p, q)}\right)$ and (5.15), we have that

$$
v\left(C P_{(p, q)}\right)=\sup _{u \in U} \inf _{x \in X}\left\{f_{u_{1}}(x-p)+g_{u_{2}}(A x+q)\right\} \leq \sup _{u \in U}\left\{f_{u_{1}}\left(p_{1}\right)+g_{u_{2}}\left(p_{2}\right)\right\} .
$$

Combing this with (5.17) and (5.18), one can see that

$$
v\left(C P_{(p, q)}\right) \leq f_{\bar{u}_{1}}\left(p_{1}\right)+g_{\bar{u}_{2}}\left(p_{2}\right)=v\left(C D_{(p, q)}\right) .
$$

This together with the weak duality implies that $v\left(C D_{(p, q)}\right)=v\left(C P_{(p, q)}\right)=v\left(C P_{(p, q)}^{\bar{u}}\right)$ and $p_{1}+$ $p$ is the optimal solution of the problem $\left(C P_{(p, q)}^{\bar{u}}\right)$.

(ii) Suppose that for each $p \in X, q \in Y$ and $\bar{u}=\left(\bar{u}_{1}, \bar{u}_{2}\right) \in U$ satisfying $y_{0}^{*} \in S(p, q ; \bar{u})$, $v\left(C D_{(p, q)}^{\bar{u}}\right)=v\left(C P_{(p, q)}^{\bar{u}}\right)$, and the problem $\left(C P_{(p, q)}^{\bar{u}}\right)$ has an optimal solution. Let $p \in X$ and $q \in Y$ be such that $A p+q \in \partial\left(f_{\bar{u}_{1}}^{*} \circ\left(-A^{*}\right)+g_{\bar{u}_{2}}^{*}\right)\left(y_{0}^{*}\right)$. Then

$$
\left\langle A p+q, y^{*}\right\rangle-f_{\bar{u}_{1}}^{*}\left(-A^{*} y^{*}\right)-g_{\bar{u}_{2}}^{*}\left(y^{*}\right) \leq\left\langle A p+q, y_{0}^{*}\right\rangle-f_{\bar{u}_{1}}^{*}\left(-A^{*} y_{0}^{*}\right)-g_{\bar{u}_{2}}^{*}\left(y_{0}^{*}\right)
$$

for each $y^{*} \in Y^{*}$,

which implies that $y_{0}^{*} \in S(p, q, \bar{u})$. Thus, by assumptions, there exists $x_{0} \in X$ such that

$$
v\left(C D_{(p, q)}^{\bar{u}}\right)=v\left(C P_{(p, q)}^{\bar{u}}\right)=f_{\bar{u}_{1}}\left(x_{0}-p\right)+g_{\bar{u}_{2}}\left(A x_{0}+q\right) .
$$


This together with (5.13) implies that

$$
f_{\bar{u}_{1}}\left(x_{0}-p\right)+g_{\bar{u}_{2}}\left(A x_{0}+q\right)=\left\langle A p+q, y_{0}^{*}\right\rangle-f_{\bar{u}_{1}}^{*}\left(-A^{*} y_{0}^{*}\right)-g_{\bar{u}_{2}}^{*}\left(y_{0}^{*}\right) .
$$

Noting the above inequality and using the definition of the conjugate function, we see that

$$
\begin{aligned}
& 0 \leq f_{\bar{u}_{1}}\left(x_{0}-p\right)+f_{\bar{u}_{1}}^{*}\left(-A^{*} y_{0}^{*}\right)+\left\langle A x_{0}-A p, y_{0}^{*}\right\rangle \text { and } \\
& g_{\bar{u}_{2}}\left(A x_{0}+q\right)-g_{\bar{u}_{2}}^{*}\left(y_{0}^{*}\right)+\left\langle A x_{0}+q, y_{0}^{*}\right\rangle \leq 0 .
\end{aligned}
$$

It follows from (5.19) that

$$
\begin{aligned}
& f_{\bar{u}_{1}}\left(x_{0}-p\right)+f_{\bar{u}_{1}}^{*}\left(-A^{*} y_{0}^{*}\right)+\left\langle A x_{0}-A p, y_{0}^{*}\right\rangle=0 \text { and } \\
& g_{\bar{u}_{2}}\left(A x_{0}+q\right)-g_{\bar{u}_{2}}^{*}\left(y_{0}^{*}\right)+\left\langle A x_{0}+q, y_{0}^{*}\right\rangle=0 .
\end{aligned}
$$

Then, by the Young equality (2.4), we have that $x_{0}-p \in \partial f_{\bar{u}_{1}}\left(-A^{*} y_{0}^{*}\right)$ and $A x_{0}+q \in \partial g_{\bar{u}_{2}}\left(y_{0}^{*}\right)$. Thus,

$$
A p+q=(-A)\left(x_{0}-p\right)+\left(A x_{0}+q\right) \in(-A) \partial f_{\bar{u}_{1}}\left(-A^{*} y_{0}^{*}\right)+\partial g_{\bar{u}_{2}}\left(y_{0}^{*}\right),
$$

and hence $A p+q \in \bigcup_{u \in U}\left[(-A) \partial f_{u_{1}}\left(-A^{*} y_{0}^{*}\right)+\partial g_{u_{2}}\left(y_{0}^{*}\right)\right]$. Therefore, by the arbitrariness of $p$ and $q$, the $(C M R)$ holds at $\left(\bar{u}_{1}, \bar{u}_{2} ; y_{0}^{*}\right)$. The proof is complete.

By Theorem 5.2, we have the following corollary directly, which extends and improves the corresponding result in [2], Theorem 6.9.

Corollary 5.1 Suppose that $f$, $g$ are proper lsc convex functions and $A$ is a linear operator. Let $y_{0}^{*} \in\left(\operatorname{dom} g^{*}\right) \cap(-A)^{*}\left(\operatorname{dom} f^{*}\right)$. Then (5.12) holds at $y_{0}^{*}$ if and only if, for each $p \in X$ and $q \in Y$ satisfying $y_{0}^{*} \in S(p, q)$, there exists $x_{0} \in X$ such that

$$
f\left(x_{0}-p\right)+g\left(A x_{0}+q\right)=\langle A p+q\rangle-f^{*}\left(-A^{*} y_{0}^{*}\right)-g^{*}\left(y_{0}^{*}\right) .
$$

\section{Competing interests}

The authors declare that they have no competing interests.

\section{Authors' contributions}

This work was carried out in collaboration between the three authors. Authors MW and DH collected the literature and wrote the first draft of the manuscript. Author $\mathrm{CZ}$ revised and improved the draft of the manuscript. All authors read and approved the manuscript.

\section{Author details}

'College of Mathematics and Statistics, Jishou University, Jishou, 416000, P.R. China. ${ }^{2}$ Business School, Sichuan University, Chengdu, 610064, P.R. China.

\section{Acknowledgements}

The authors are grateful to both anonymous reviewers for valuable suggestions and remarks which helped to improve the quality of the paper. The first and the second authors were supported in part by the National Natural Science Foundation of China (grant 11461027) and the Scientific Research Fund of Hunan Provincial Education Department (grant 13B095), the third author was supported in part by the National Natural Science Foundation of China (grant 71471122). 


\section{References}

1. Boț, Rl, Grad, SM, Wanka, G: Duality in Vector Optimization. Springer, Berlin (2009)

2. Li, C, Fang, DH, López, G, López, MA: Stable and total Fenchel duality for convex optimization problems in locally convex spaces. SIAM J. Optim. 20, 1032-1051 (2009)

3. Zălinescu, C: Convex Analysis in General Vector Spaces. World Scientific, Singapore (2002)

4. Borwein, JM, Jeyakumar, V, Lewis, A, Wolkowicz: Constrained approximation via convex programming. Preprint. University of Waterloo, Waterloo, Ontario (1988)

5. Holmes, RB: Geometric Functional Analysis. Springer, New York (1993)

6. Rockafellar, RT: Conjugate Duality and Optimization. Conference Board of the Mathematical Sciences Regional Conference Series in Applied Mathematics, vol. 16. SIAM, Philadelphia (1974)

7. Burachik, RS, Jeyakumar, V, Wu, ZY: Necessary and sufficient conditions for stable conjugate duality. Nonlinear Anal. 64, 1998-2006 (2006)

8. Ben-Tal, A, Nemirovski, A: Robust optimization-methodology and applications. Math. Program., Ser. B 92, 453-480 (2002)

9. Ben-Tal, A, Nemirovski, A: A selected topics in robust convex optimization. Math. Program., Ser. B 112, 125-158 (2008)

10. Beck, A, Ben-Tal, A: Duality in robust optimization: primal worst equals dual best. Oper. Res. Lett. 37, 1-6 (2009)

11. Jeyakumar, V, Li, GY: Strong duality in robust convex programming: complete characterizations. SIAM J. Optim. 20 3384-3407 (2010)

12. Jeyakumar, V, Li, GY: Characterizing robust set containments and solutions of uncertain linear programs without qualifications. Oper. Res. Lett. 38, 188-194 (2010)

13. Jeyakumar, V, Li, GY: Robust Farkas lemma for uncertain linear systems with applications. Positivity 15, 331-342 (2011)

14. Ben-Tal, A, Ghaoui, LE, Nemirovski, A: Robust Optimization. Princeton Series in Applied Mathematics (2009)

15. Jeyakumar, V, Li, GY, Wang, JH: Some robust convex programs without a duality gap. J. Convex Anal. 20, 377-394 (2013)

16. Li, GY, Jeyakumar, V, Lee, GM: Robust conjugate duality for convex optimization under uncertainty with application to data classification. Nonlinear Anal. 74, 2327-2341 (2011)

17. Ben-Tal, A, Nemirovski, A, Roos, C: Robust solutions of uncertain quadratic and conic-quadratic problems. SIAM J. Optim. 13(2), 535-560 (2002)

18. Bertsimas, D, Brown, D: Constructing uncertainty sets for robust linear optimization. Oper. Res. 57, 1483-1495 (2009)

19. Bertsimas, D, Pachamanova, D, Sim, M: Robust linear optimization under general norms. Oper. Res. Lett. 32, 510-516 (2004)

20. Jeyakumar, V, Li, GY: New dual constraint qualifications characterizing zero duality gaps of convex programs and semidefinite programs. Nonlinear Anal. 71, 2239-2249 (2009)

21. Li, C, Zhao, XP, Hu, YH: Quasi-Slater and Farkas-Minkowski qualifications for semi-infinite programming with applications. SIAM J. Optim. 23(4), 2208-2230 (2013)

22. Li, C, Ng, KF, Pong, TK: Constraint qualifications for convex inequality systems with applications in constrained optimization. SIAM J. Optim. 19, 163-187 (2008)

23. Phelps, RR: Convex Functions, Monotone Operators and Differentiability, 2nd edn. Lecture Notes in Mathematics, vol. 1364. Springer, Berlin (1993)

\section{Submit your manuscript to a SpringerOpen ${ }^{\circ}$ journal and benefit from:}

- Convenient online submission

- Rigorous peer review

- Immediate publication on acceptance

- Open access: articles freely available online

- High visibility within the field

- Retaining the copyright to your article 